\title{
Theoretical Evidence for Wave Nature of Micro Particle and New Theory of Its Collective Motion in Material
}

\author{
Takahisa Okino \\ Department of Applied Mathematics, Faculty of Science \& Engineering, Oita University, Oita, Japan \\ Email: okino@oita-u.ac.jp
}

How to cite this paper: Okino, T. (2021) Theoretical Evidence for Wave Nature of Micro Particle and New Theory of Its Collective Motion in Material. Journal of Modern Physics, 12, 260-283.

https://doi.org/10.4236/jmp.2021.123020

Received: January 2, 2021

Accepted: February 20, 2021

Published: February 23, 2021

Copyright $\odot 2021$ by author(s) and Scientific Research Publishing Inc. This work is licensed under the Creative Commons Attribution International License (CC BY 4.0).

http://creativecommons.org/licenses/by/4.0/

\section{(c) (i) Open Access}

\begin{abstract}
Since a material is composed of micro particles, investigating behavior of those particles is essentially dominant for materials science. The diffusivity of diffusion equation is relevant to not only a collective motion of micro particles but also a motion of single particle. An elementary process of diffusion was thus theoretically investigated in a local space and time. As a result, the investigation concluded that the wave nature of micro particle results from denying the mathematical density theorem of a real time in the Newton mechanics. In other words, the basic theory of quantum mechanics is established in accordance with the cause-and-effect relationship in the Newton mechanics, for the first time, regardless of the de Broglie hypothesis. In relation to the collective motion of micro particles, the new diffusion theory was also reasonably established using the universal expression of diffusivity obtained here. In the present paper, the new findings indispensable for the fundamental knowledge in physics are thus systematically discussed in accordance with the theoretical frame in physics.
\end{abstract}

\section{Keywords}

Diffusion, Fick's Laws, de Broglie’s Hypothesis, Schrödinger's Equation, Matter-Wave

\section{Introduction}

There is occasionally an important relation in natural phenomena where it is universally valid under a given condition. When we cannot reveal the theoretical evidence, it has been accepted as a law or a principle in physics. Further, the equation derived theoretically from its law or principle has been accepted as a basic 
one in physics, where it corresponds to a theorem in mathematics. In accordance with such theoretical frame, physics has developed until now.

The basic theory in physics lies in Newton's laws established under the condition of having the common time between arbitrary coordinate systems. Einstein's relativity [1], which is one of the modern physics, was established in 1905 by denying the absolute time in the Newton mechanics in accordance with the constant principle of light speed $c$. However, Newton's laws are still acceptable as dominant ones in physics under the condition of $v \ll c$, where $v$ is a speed of a body with mass $m$.

On the other hand, the quantum theory, which is the other modern physics, was established by accepting the de Broglie hypothesis [2] of

$$
m v=p=h / \lambda,
$$

where $h, \lambda$ are the Planck constant [3] and a wave length of matter-wave defined here. In the Newton mechanics, both the particle nature and wave one are not simultaneously accepted. The conception of matter-wave is thus extremely novel. We had not understood the cause-and-effect relationship between the Newton mechanics and the quantum mechanics until recently. In the following, it will be thus revealed that the quantum mechanics is theoretically established by denying the mathematical density theorem of a real time in the Newton mechanics.

In 1923, de Broglie assumed that the result $m c^{2}$ of Einstein's relativity and the photon energy $h v$ of the Planck theory relevant to a light of frequency $v$ are equivalent to each other. Further, he assumed that $c / v$ corresponds to $\lambda$ of a matter-wave, if we accept the replacement of $c \rightarrow v$ in the relation $m c=h v / c$ obtained here. After that, the experimental results revealed that an electron has an intrinsic nature like a wave [4]. In relatively recent years, the experimental results revealed that an atom or a molecule has also an intrinsic nature like a wave [5]. However, we did not confirm whether such a micro particle satisfies Equation (1) or not, even if it had an intrinsic nature like a wave. Further, we did not understand the theoretical evidence that a micro particle in the Newton mechanics has a wave nature.

For a micro particle with mass $m$, the wave equation $\Psi(t, x, y, z)$ given by

$$
i \hbar \frac{\partial}{\partial t} \Psi(t, x, y, z)=-\frac{\hbar^{2}}{2 m}\langle\tilde{\nabla} \mid \nabla\rangle \Psi(t, x, y, z)
$$

was derived by Schrödinger in 1926 from Equation (1) [6]. It was revealed that the Schrödinger Equation (2) is applicable to behavior of an arbitrary micro particle. This indicates indirectly that Equation (1) is valid for those micro particles. As far as Equation (2) is derived from Equation (1), however, understanding of the correlation between the quantum mechanics and the Newton mechanics is essentially impossible. Therefore, the correlation between them has been understood as an afterthought for a long time by using the correspondence principle between a physical quantity in the Newton mechanics and the corresponding operator in the quantum mechanics. From a viewpoint of the theoretical frame in the fundamental physics, therefore, the quantum theory is still incom- 
plete, as far as the cause-and-effect relationship between the quantum mechanics and the Newton mechanics is not revealed.

For a collective motion of micro particles in the space-time $(t, x, y, z)$, Fick's first law relevant to the diffusion flux $\left|J_{\mathrm{F}}\right\rangle$ given by

$$
\left|J_{\mathrm{F}}\right\rangle=-D|\nabla\rangle C
$$

was proposed in 1855 , where $C(t, x, y, z)$ and $D$ are a concentration of micro particles and a diffusivity [7]. Fick's second law was accepted as a nonlinear partial differential equation of

$$
\Omega C=0, \text { for } \Omega=\frac{\partial}{\partial t}-\langle\tilde{\nabla} \mid D \nabla\rangle,
$$

where $\langle\tilde{\nabla}|=-| \nabla\rangle^{\dagger}$ is used because of the Hermite conjugate of the Dirac bracket for a differential operator. In physics, Equation (3) shows that the diffusivity $D$ is a proportional factor of a concentration gradient to the diffusion flux $\left|J_{\mathrm{F}}\right\rangle$. In mathematics, Equation (4) shows that $D$ is an operator in the operator $\Omega$. As far as we accept Equations (3) and (4), therefore, we cannot understand the physical essence of $D$ from their equations.

Here, the new findings are as follows.

1) The diffusion equation, having been accepted as the Fick second law since 1855, was theoretically derived from the mathematical theory of Markov's process [8] [9] [10]. It was then found that the elementary quantity of diffusivity $D$ corresponds to the angular momentum and is expressed as $D=\hbar / 2 m$ [11] [12] [13]. The universal expression of diffusivity, which is applicable to any diffusion problem, was also derived.

2) The diffusion equation using $D=\hbar / 2 m$ for a micro particle in an isolated local space was transformed into the wave Equation (2) of Schrödinger by denying the mathematical density theorem of a real time in the Newton mechanics [11] [12] [13]. The wave nature of an arbitrary micro particle was, for the first time, theoretically revealed in accordance with the cause-and-effect relationship between the quantum mechanics and the Newton mechanics. Further, it was theoretically revealed that Equation (1) of matter-wave is not a hypothesis but a basic equation in physics [12] [13].

3) The general solutions of nonlinear diffusion equation, which had never been solved since 1855, were reasonably obtained [14]. As a result, the new diffusion theory of a multi-components system was reasonably established in relation to the transformation from a diffusion equation of a moving coordinate system into that of a fixed one, and vice versa [9] [10].

As far as a material is composed of micro particles, investigating behavior of those particles is indispensable for research subjects in the materials science. In the following, it will be theoretically confirmed that the theory of diffusion plays an important role for fundamental problems in the materials science.

\section{Theoretical Frame in Classical Quantum Theory}

From a viewpoint of theoretical frame in physics, fundamental problems in the 
classical quantum theory are briefly discussed in accordance with a time series in the following.

In the space-time $(t, x, y, z)$, the Markov process [8] in mathematics is applicable to such behavior as a collective motion of micro particles in an isolated physical system. As a result, the diffusion equation of

$$
\frac{\partial}{\partial t} C(t, x, y, z)=D\langle\tilde{\nabla} \mid \nabla\rangle C(t, x, y, z)
$$

was theoretically obtained as a nonlinear partial differential equation [9] [10]. In the isolated local space, the diffusivity $D$ relevant to a micro particle with mass $m$ is then obtained as

$$
D=\frac{(\Delta r)^{2}}{2 \Delta t}=\frac{\Delta r p}{2 m}, \text { for } r=\sqrt{x^{2}+y^{2}+z^{2}},
$$

where $p(=m v)$ is a momentum of micro particle. Equation (6) shows that the diffusivity satisfies the relation of parabolic law. Further, a micro particle in the isolated local space has an angular momentum because of the term $\Delta r p$. In other words, the micro particle makes a circuit on the surface of isolated local space. This corresponds to phenomena known as a lattice vibration or a thermal vibration of atoms in a material.

The diffusion Equation (5) derived here is a moving coordinate system judging from the derivation process. It will be revealed that we can transform it into the relation (4) of a fixed coordinate system, and vice versa. It will be also revealed that the diffusivity expression obtained here plays a complementary role for incompletion of the theoretical frame in the quantum theory.

As seen from the atomic hypothesis of Dalton in 1803 and the law of Avogadro in 1811, the chemists in those days thought that a material is composed of atoms or molecules as fundamental particles. On the other hand, the velocity distribution function of Maxwell in 1859 or Boltzmann in 1968 was reported in physics as a problem of mechanical elastic collision between these fundamental particles. The theoretical frame developed here was relevant to a theory between the thermodynamics and the Newton mechanics, where the averaged impulse resulting from collisions between micro particles corresponds to a thermodynamic pressure of macro quantity in physics. Further, the equipartition of energy reported in 1876 was the theory that a mechanical energy of micro particle corresponds to an absolute temperature of macro quantity in the thermodynamic state [15].

By assuming a micro particle as a component of material, it seemed in those days that the equipartition of energy reveals what a material is composed of fundamental particles. However, it was found that the equipartition of energy cannot explain the theory of specific heat in a low temperature region. In the end of 19 century, therefore, there was no such firm theory in physics that a material is composed of atoms and/or molecules as fundamental particles.

In circumstances mentioned above, Planck in 1900 [3] reported the theoretical relation indicating what the light of electromagnetic wave has a nature like a 
particle. We cannot originally accept a picture having simultaneously a particle nature and a wave one in the classical physics. After that, however, Einstein in 1905 [16] and Compton in 1923 [17] revealed that the light has both a wave nature and a particle one.

On the other hand, in relation to the Brown motion relevant to a random movement of pollen in water, Einstein in 1905 [18] revealed that the self-diffusion phenomena of water molecules are visualized by behavior of pollen. In other words, the relation of diffusivity obtained here shows that a material is composed of atoms and/or molecules as fundamental particles. As mentioned later in a head of Einstein's paradox, however, there is a problem in the theoretical frame developed then. In addition, Langevin in 1908 [19] also derived a similar relation to Einstein's theory from analyzing an equation of motion for a micro particle.

In accordance with the empirical equation of radiant light reported by Balmer in 1885 [20], Bohr in 1913 [21] proposed a model of atomic structure under the quantum condition and the frequency condition. In the model, an electron moves on a specific circular orbit $r_{n}(n=1,2, \cdots)$ around the nucleus and it jumps from an orbit to an adjacent orbit through the radiation or absorption of energy $\Delta E=h v$. Using a momentum $p$ of electron, the notation $\Delta r=r_{n}-r_{n-1}\left(r_{0}=0\right)$ and the angular momentum $|L\rangle$ yielding

$$
|L\rangle=|r \times p\rangle \rightarrow|\Delta L\rangle=\left|r_{n} \times p_{n}\right\rangle-\left|r_{n-1} \times p_{n-1}\right\rangle, \quad \Delta L=\sqrt{\langle\Delta L \mid \Delta L\rangle},
$$

the quantum condition is rewritten as

$$
\Delta L=\Delta r p=\hbar .
$$

After the Bohr model, the experimental results of Frank and Hertz in 1914 [22] suggested that an electron of the Bohr model moves on a specific circular orbit around the nucleus concerned. Further, it was experimentally revealed that the electronic beam has diffraction phenomena of a wave characteristic [4]. Based on the experimental results, it is considered that an electron of the Bohr model has a wave nature as a matter-wave. When an electron of the Bohr model satisfies the relation of

$$
\lambda=2 \pi \Delta r,
$$

it moves stably on a specific circular orbit as a stationary wave. Here, if we eliminate $\Delta r$ from Equations (7) and (8), Equation (1) reasonably obtained.

It was thus found that Equation (1) is valid for an electron of the Bohr model. Here, we think the function $\varphi$ of progressive wave in the space-time $(t,|r\rangle)$ given by

$$
\varphi=A \exp [i(\langle k \mid r\rangle-\omega t)],
$$

where the notations $A,\langle k|$ and $\omega$ are an amplitude of vibration of a frequency $v$, a wave number vector of $k=\sqrt{\langle k \mid k\rangle}=2 \pi / \lambda$ relevant to a wave length $\lambda$ and angular velocity of $\omega=2 \pi v$. In that case, Equations (1), (7) and (8) satisfy the relation $k=2 \pi / \lambda=1 / \Delta r$ relevant to the progressive wave then. It was thus 
confirmed that Equation (1) is valid also for a motion of free electron. At this point, as far as we discuss the motion of an electron, Equation (1) is not a hypothesis but a basic equation in accordance with the theoretical frame in physics.

In circumstances mentioned above, de Broglie in 1923 [2] proposed Equation (1) as a hypothesis applicable to an arbitrary micro particle. After that, Schrödinger in 1926 [2] derived a wave Equation (2) from using Equation (1). It was found that the Schrödinger equation is applicable to behavior of an arbitrary micro particle. The Schrödinger equation is directly derived from the relation of matter-wave then. The theoretical evidence that a micro particle in the Newton mechanics has a wave nature has never been revealed since 1926. The theoretical frame of the quantum mechanics is thus still incomplete without revealing the causality for the Newton mechanics.

\section{Consistency of Quantum Theory with Basic Theory in Physics}

The basic idea in physics lies in the Newton mechanics. For example, Einstein's relativity was established by denying the absolute time in the Newton mechanics. In the following, it will be revealed that we can establish the quantum theory by denying the mathematical density theorem of a real time in the Newton mechanics.

As a formal problem between Equations (2) and (5), if we rewrite $D \rightarrow i \hbar / 2 m$ and $C \rightarrow \hbar \Psi$ in Equation (5), it is transformed into Equation (2). If the transformation from the diffusion equation relevant to a picture of micro particles into the wave equation of Schrödinger relevant to a wave picture is theoretically possible, we can then obtain the evidence that a micro particle in the Newton mechanics has a wave nature. In history, therefore, the transformation from Equation (5) into Equation (2) was investigated by the giant figures in physical society like Einstein, Bohm and others. However, the attempt was not successful. It is considered that the root cause of failure lies in having accepted the diffusion equation as a law of Fick without physical essence of diffusivity.

Here, the Fick second law shows that the diffusivity is related to a micro particle in the diffusion region. Nevertheless, we cannot grasp the physical essence of diffusivity relevant to a micro particle from the Fick laws. About that matter, as mentioned above, Okino began by deriving the diffusion equation from the mathematical theory of Markov process in order to grasp the physical essence of diffusivity. In the following, the fundamental theory in physics will be developed using Equation (6) obtained then.

Applying the equipartition of energy to a free electron in a material gives the relation of

$$
\Delta r p=\alpha\left(k_{\mathrm{B}} T+\varepsilon\right) \Delta t,
$$

where $k_{\mathrm{B}}, \varepsilon$ and $\alpha$ are the Boltzmann constant, a correction term for the uncertain principle at $T=0$ and a degree of freedom of micro particle, for example, $\alpha=3$ in case of a mono-atomic molecule. Since the free electron satisfies 
also the relation of $\Delta r p=\hbar, \Delta t\left(=\hbar / \alpha\left(k_{\mathrm{B}} T+\varepsilon\right)\right)$ depends only on an absolute temperature. Here, note that there is no characteristic quantity of a free electron in Equation (10) in spite of the discussion about the free electron itself. This means that Equation (10) is valid also for an arbitrary micro particle in a material [13] when the equipartition of energy is applied to it. It is, therefore, revealed that Equation (7) of

$$
\Delta L=\Delta r p=\hbar
$$

becomes also valid for an arbitrary micro particle in a material [11] [12] [13].

By substituting Equation (7) into Equation (6), the elementary quantity of diffusivity yielding

$$
D=\frac{\Delta L}{2 m}=\frac{\hbar}{2 m}
$$

is obtained in the isolated local space. In the following, the wave nature of an arbitrary micro particle will be revealed using Equation (11).

When $d$ is a distance between two micro particles $\mathrm{A}$ and $\mathrm{B}$ of the same kind in the isolated local space, it is necessary to observe a reflected light of wave length $\lambda<d$ for the discrimination of A from B. If $d$ is very small, a high energy of $\hbar c / \lambda$ is necessary for the discrimination. As a result, we cannot then discriminate them because of a turbulence caused by the high energy. In that situation, we consider an elastic collision problem between the above micro particle $\mathrm{A}$ and $\mathrm{B}$ in the following, where the particle A moves with a velocity $v_{\mathrm{A}}=v_{0}$ and the particle $\mathrm{B}$ is in the rest state of the velocity $v_{\mathrm{B}}=0$.

If we can identify the difference between the micro particles $A$ and $B$ in the space and time given by $\left(\Delta t_{\mathrm{A}}, \Delta r_{\mathrm{A}}\right)$ and $\left(\Delta t_{\mathrm{B}}, \Delta r_{\mathrm{B}}\right)$, the particle $\mathrm{A}$ reaches $v_{\mathrm{A}}=0$ from a velocity $v_{\mathrm{A}}=v_{0}$ with the acceleration $a_{\mathrm{A}}=-\infty$ because of $\lim _{\Delta t_{\mathrm{A}} \rightarrow 0}\left\{\Delta r_{\mathrm{A}} /\left(\Delta t_{\mathrm{A}}\right)^{2}\right\}$ and subsequently the particle B reaches $v_{\mathrm{B}}=v_{0}$ from the velocity $v_{\mathrm{B}}=0$ with the acceleration $a_{\mathrm{B}}=\infty$ because of $\lim _{\Delta t_{\mathrm{B}} \rightarrow 0}\left\{\Delta r_{\mathrm{B}} /\left(\Delta t_{\mathrm{B}}\right)^{2}\right\}$. On the other hand, if we cannot identify the difference between them, it seems that the particle A reaches $v_{\mathrm{A}}=0$ from a velocity $v_{\mathrm{A}}=v_{0}$ with the acceleration $a_{\mathrm{A}}=-\infty$ and subsequently the particle A reaches $v_{\mathrm{A}}=v_{0}$ again from the velocity $v_{\mathrm{A}}=0$ with the acceleration $a_{\mathrm{A}}=\infty$. For the behavior of the particle $\mathrm{A}$ in the collision time $\Delta t_{\mathrm{C}}$ between $0<\Delta t_{\mathrm{C}}<\Delta t_{\mathrm{A}}+\Delta t_{\mathrm{B}}$, the acceptance of $a_{\mathrm{A}}=\infty$ between $0<\Delta t<\Delta t_{\mathrm{B}}$ indicates that we must accept the relation of $\Delta t \rightarrow i \Delta t$ in accordance with the definition of acceleration $\lim _{\Delta t \rightarrow 0}\left\{\Delta r /(\Delta t)^{2}\right\}$, resulting from the impossibility of the discrimination of A from B between $0<\Delta t<\Delta t_{\mathrm{B}}$ [11] [12] [13].

The matter mentioned here is equivalent to denying the mathematical density theorem of a real time in the Newton mechanics. In other words, there is a minimum unit time $\varepsilon_{t}$ as a real time and the relation of $\Delta t \rightarrow i \Delta t$ is reasonably acceptable in the region of $\Delta t<\varepsilon_{t}$ [11]. Generally, as can be seem from rewriting a partial differential equation into the difference equation, it means a re- 
lation valid in a local space-time $(\Delta t,\langle\Delta r|)=(\Delta t, \Delta x, \Delta y, \Delta z)$. The relation of $\Delta t \rightarrow i \Delta t$ is thus valid only in such a local space-time. If $t \rightarrow i t$ is valid, the general solution of partial differential equation becomes not a complex value function but a complex function then.

In accordance with the discussion mentioned above, accepting the impossibility of discrimination between two particles of the same kind in a local space corresponds to accepting the relation of differential operator given by

$$
\frac{\partial}{\partial t}=\lim _{\Delta t \rightarrow 0} \frac{\Delta}{\Delta t} \rightarrow \lim _{\Delta t \rightarrow 0} \frac{\Delta}{ \pm i \Delta t}=\mp i \frac{\partial}{\partial t}
$$

in the present theory. It was revealed that there is no conception of acceleration for a motion of micro particle in a local space [13]. As shown in the relation of matter-wave, however, the conception of velocity is still valid in a local space. Therefore, judging from the correlation between differential operators expressed by

$$
\frac{1}{v_{x}}=\lim _{\Delta t \rightarrow 0} \frac{\Delta t}{\Delta x}=\frac{\partial}{\partial x} t \rightarrow \lim _{\Delta x \rightarrow 0} \frac{ \pm i \Delta t}{\Delta x}= \pm i \frac{\partial}{\partial x} t,
$$

the relation of differential operator of

$$
\frac{\partial}{\partial x} \rightarrow \pm i \frac{\partial}{\partial x}
$$

should be consequently valid. It will be found that the imaginary operator shown here corresponds to a real eigenvalue of the Hermite operator. In addition, as seen from the above discussion, note that a local space is clearly real and $\Delta r \rightarrow \pm i \Delta r$ is not valid then.

A plus or a minus sign of the above imaginary operators is determined from eigenvalues of Equation (9). When $\pm i|\nabla\rangle$ operates on Equation (9), we determine $|\nabla\rangle \rightarrow-i|\nabla\rangle$ like the obtained eigenvalue corresponds to the direction of movement of progressive wave. In a similar manner, we determine $\frac{\partial}{\partial t} \rightarrow i \frac{\partial}{\partial t}$. Thus, the differential operators in the Newton mechanics corresponds to

$$
\left\{\begin{array}{l}
\frac{\partial}{\partial t} \rightarrow i \frac{\partial}{\partial t} \\
|\nabla\rangle \rightarrow-i|\nabla\rangle
\end{array}\right.
$$

in the quantum mechanics in accordance with the causality [11] [12] [13].

Substituting Equations (11) and (12) into Equation (5) yields the Schrödinger Equation (2) by rewriting $C \rightarrow \hbar \Psi$. In this case, it is revealed that $\Psi$ becomes a complex value function then [11]. Judging from the transformation of the diffusion equation of micro particles into the wave equation of Schrödinger, at this point, it was theoretically revealed that an arbitrary micro particle has a wave nature. Therefore, Equation (8) of

$$
\lambda=2 \pi \Delta r
$$

is valid not only for a free electron but also for an arbitrary micro particle. 
Hereinbefore, since it was theoretically revealed that Equations (7) and (8) are valid for an arbitrary micro particle, eliminating $\Delta r$ from their equations yields the relation of matter-wave expressed by Equation (1). In accordance with the theoretical frame in physics, at this point, Equation (1) proposed by de Broglie as a hypothesis is now not a hypothesis but a basic equation in physics. Hereafter, the relation of matter-wave should not be thus named a hypothesis except the historical description.

In the above theoretical development, for a micro particle in a material, the relation of

$$
\lambda=h / \sqrt{\alpha m\left(k_{B} T+\varepsilon\right)}
$$

was obtained as a new expression of matter-wave [13]. It is considered that Equation (13) is applicable to the diffusion theory based on the matter-wave. For example, when the averaged distance between micro particles in a material is expressed as $2 a$, a micro particle under the condition of $m<\hbar^{2} / \alpha a^{2}\left(k_{\mathrm{B}} T+\varepsilon\right)$ cannot exist in a local space and it moves through interstices between micro particles while repeating collisions with other micro particles. As can be seen from the definition of diffusivity given by Equation (6), the behavior of such micro particles as satisfying $m<\hbar^{2} / \alpha a^{2}\left(k_{\mathrm{B}} T+\varepsilon\right)$ should be expressed not by diffusion Equation (5) but by the wave equation expanded into the many-body problem in the quantum mechanics. As an example, the tunnel effect is known as such a case if the number of collision times is too few in a thin film.

In the past, the correlation between the quantum mechanics and the Newton mechanics has been discussed as an afterthought in accordance with the correspondence principle between an operator in the quantum mechanics and the corresponding physical quantity in the Newton mechanics. For example, the relations of a momentum $p$ and energy $E$ in the Newton mechanics have been accepted as

$$
\left\{\begin{array}{l}
p=-i \hbar \nabla \\
E=i \hbar \frac{\partial}{\partial t}
\end{array}\right.
$$

in the quantum mechanics. In accordance with the causality for the Newton mechanics discussed above, Equation (12) shows that Equation (14) is reasonably derived as

$$
\left\{\begin{array}{l}
p_{x}=h / \lambda_{x}=\frac{h}{2 \pi \Delta x} \rightarrow \lim _{\Delta x \rightarrow 0} \hbar \frac{\Delta}{\Delta x}, \quad p_{x}=\hbar \frac{\partial}{\partial x} \rightarrow p_{x}=-i \hbar \frac{\partial}{\partial x} \\
E=h v=\frac{h}{2 \pi \Delta t} \rightarrow \lim _{\Delta t \rightarrow 0} \hbar \frac{\Delta}{\Delta t}, E=\hbar \frac{\partial}{\partial t} \rightarrow E=i \hbar \frac{\partial}{\partial t}
\end{array}\right.
$$

from $p_{x}=h / \lambda_{x} \quad\left(\lambda_{x}=2 \pi \Delta x\right)$ and $E=h v, \quad(v=1 / 2 \pi \Delta t)$ in the present theory.

\section{Importance of Derivation of Diffusion Equation}

Hereinbefore, it was found that the theory of elementary process of diffusion 
plays a dominant role for understanding a physical essence having been disregarded in the classical quantum theory. On the other hand, analyzing a diffusion equation relevant to a collective motion of micro particles has been widely performed as a basic theory in the materials science. In that situation, there are extremely serious misunderstanding problems in the existing diffusion theory having been widely accepted for a long time [23] [24]. In the following, therefore, the new diffusion theory will be developed in accordance with the general theory in the mathematical physics [9] [10].

1) Theoretical frame of Fick's laws

Fick proposed in 1855 that the thermal conduction equation derived by Fourier in 1822 is applicable to the diffusion phenomena for a collective motion of micro particles [7]. In that case, the Fick first law of diffusion flux $\left|J_{\mathrm{F}}\right\rangle$ corresponding to the thermal flux of Fourier was then defined as Equation (3) of

$$
\left|J_{\mathrm{F}}\right\rangle=-D|\nabla\rangle C(t, x, y, z) \text {. }
$$

And the Fick second law of diffusion equation in a broad sense, corresponding to the thermal conduction equation of Fourier, was defined as Equation (4) of

$$
\partial_{t} C(t, x, y, z)=\langle\tilde{\nabla} \mid D \nabla\rangle C(t, x, y, z) .
$$

It seems that the reason for the proposal of laws in those days results from the realization of parabolic law shown in experimental profiles of distribution relevant to a concentration as well as a temperature in a material.

When Fick's laws were proposed, Gauss's diversion theorem was already reported in 1813. As shown in the following, therefore, judging from the theoretical frame in physics, it is inadequate that we accept each of equations (3) and (4) as an independent law.

For a differentiable spatial vector $|A(x, y, z)\rangle$ in a region $V$ within a single closed surface $S$, Gauss's diversion theorem shows that the correlation between a volume integral and a surface integral yielding

$$
\iiint_{V}\langle\tilde{\nabla} \mid A\rangle \mathrm{d} V=\iint_{S}\langle n \mid A\rangle \mathrm{d} S
$$

is valid, where $\langle n|$ is a unit vector perpendicular to a surface element $\mathrm{d} S$.

When Gauss's diversion theorem is applied to a flux vector $|J(t, x, y, z)\rangle$ relevant to a physical quantity $C(t, x, y, z)$ per a unit volume in the region $V$, as far as there is no sink and source of $C(t, x, y, z)$ in the region $V$, the relation of

$$
\iint_{S}\langle n \mid J(t, x, y, z)\rangle \mathrm{d} S=-\iiint_{V} \frac{\partial}{\partial t} C(t, x, y, z) \mathrm{d} V
$$

is physically valid in relation to the law of material conservation. Equations (15) and (16) yield the well-known continuous equation given by

$$
\frac{\partial}{\partial t} C(t, x, y, z)+\langle\tilde{\nabla} \mid J(t, x, y, z)\rangle=0 .
$$

Here, we should mathematically consider a degree of freedom for a diffusion flux because of $\langle\tilde{\nabla} \| J(t)\rangle=\left\langle\tilde{\nabla} \| J_{\text {eq }}\right\rangle=0$ for $|J(t)\rangle$ dependent only on a time 
$t$ and $\left|J_{\text {eq }}\right\rangle$ independent of the space-time. The diffusion flux should be thus expressed as

$$
|J(t, x, y, z)\rangle=\left|J_{\mathrm{F}}\right\rangle+|J(t)\rangle+\left|J_{\text {eq }}\right\rangle .
$$

In the following, it will be revealed that $|J(t)\rangle$ and $\left|J_{\text {eq }}\right\rangle$ are indispensable for understanding diffusion phenomena [9] [10].

If we substitute Equations (3) or (18) into Equation (17), Equation (4) is reasonably obtained. Judging from the theoretical frame in physics, therefore, Equation (4) is not a law but a basic equation then, if we accept Equations (3) or (18) as a law. However, Fick's first law of Equation (3) is unsuitable as a universal law because of $|J(t)\rangle=\left|J_{\text {eq }}\right\rangle=0$. On the other hand, if we accept Equation (4) as a law, Equations (3) or (18) is obtained by comparing it with Equation (17). In this case, Equations (3) or (18) is a basic equation judging from the theoretical frame in physics.

As mentioned above, the only diffusion Equation (4) would be accepted as a law relevant to diffusion phenomena in those days. At present, however, the diffusion Equation (4) is also not a law but a basic equation in physics because of the theoretical derivation [9] [10].

\section{2) Theoretical expression of diffusivity}

The diffusion Equation (5) was theoretically derived as a basic equation of moving coordinate system from a behavior of micro particle in an isolated local space. The diffusivity obtained here was expressed as Equation (6) then, and further it was also expressed as Equation (11) resulting from applying the equipartition of energy to a free electron in a material. In general, each of diffusion particles in local spaces is physically different conditions from each other. In a case where Equations (4) or (5) is applied to a whole diffusion region, we must consider a dependence of the space-time $(t, x, y, z)$ on the diffusivity. However, a thermal effect on a diffusion particle in a material and a mechanical interaction between a diffusion particle and the surrounding other micro particles have not been incorporated into Equations (6) or (11) yet.

For a micro particle in a state of activation energy $Q$ in a material at a temperature $T$, we incorporate the Boltzmann factor $\exp \left[-Q / k_{\mathrm{B}} T\right]$ relevant to an existence probability of the diffusion particle into Equation (11) [25]. When a micro particle interacts with the surrounding other particles, we also incorporate the potential energy $U$ of an external force $|F\rangle$ operating on the micro particle into the Boltzmann factor. The universal expression of diffusivity is thus expressed as

$$
D=\frac{\hbar}{2 m} \exp \left[\frac{U-Q}{k_{\mathrm{B}} T}\right], U=-\int F \mathrm{~d} r \text { for } F \mathrm{~d} r=\langle F \mid \mathrm{d} r\rangle .
$$

Judging from Equation (6) in a local space, a jumping velocity $\left|v_{\mathrm{p}}\right\rangle$ of a micro particle from a local space to another one is obtained as a diffusivity gradient of

$$
\left|v_{\mathrm{p}}\right\rangle=|\nabla\rangle D=-\frac{|F\rangle}{k_{\mathrm{B}} T} D .
$$


When there are $N$ elements in a diffusion region, we assume a concentration $C^{j}$ of a $j(=1,2, \cdots, N)$ element, which is normalized by total number of diffusion particles. Rewriting the concentration and diffusivity of equation (4) into $C^{j}$ and $D^{j}$, the diffusion equation of a $j$ element becomes

$$
\frac{\partial}{\partial t} C^{j}(t, x, y, z)=\left\langle\tilde{\nabla} \mid D^{j} \nabla\right\rangle C^{j}(t, x, y, z)
$$

where the normalized condition of $\sum_{j=1}^{N} C^{j}=1$ is valid, as far as there is no sink and source in the diffusion region.

For a $k$ element among $N$ elements in the diffusion region, the relation of

$$
\sum_{j=1}^{N}\left\langle\tilde{\nabla}\left|\left(D^{j}-D^{k}\right)\right| \nabla\right\rangle C^{j}(t, x, y, z)=0
$$

is valid because of the normalized condition of $\sum_{j=1}^{N} C^{j}=1$. Since the term $|\nabla\rangle C^{j}$ in Equation (22) is linearly independent of each other, the relation of $D^{j}-D^{k}=0$ is mathematically valid in Equation (22). In other words, the relation of

$$
\tilde{D}=D^{1}=\cdots=D^{j}=\cdots=D^{N}
$$

is thus valid in interdiffusion problems for a $N$ elements system. Here, note that Equation (23) means not a relation of physical diffusivities but that of mathematical operators valid only in Equation (21) [9] [10].

Equation (21) is thus rewritten as

$$
\frac{\partial}{\partial t} C^{j}(t, x, y, z)=\langle\tilde{\nabla} \mid \tilde{D} \nabla\rangle C^{j}(t, x, y, z) .
$$

As a matter of course, when we substitute initial and/or boundary values of a $j$ element into the general solution $\tilde{D}$ of Equation (24), Equation (23) shows that the physical solution $D^{j}$ is then obtained by the replacement of $\tilde{D} \rightarrow D^{j}$ and $D^{j} \neq D^{k}$ is valid if $j \neq k$.

\section{3) General solutions of nonlinear diffusion equation}

The general solutions of nonlinear diffusion equation had never been reported in the history of diffusion. In other words, even general solutions of the onedimensional space yielding

$$
\frac{\partial}{\partial t} C(t, x)=\frac{\partial}{\partial x}\left\{D \frac{\partial}{\partial x}\right\} C(t, x)
$$

had been impossible. In 1894, Boltzmann transformed Equation (25) into the nonlinear ordinary differential equation of

$$
-\frac{\xi}{2} \frac{\mathrm{d} C}{\mathrm{~d} \xi}=\frac{\mathrm{d}}{\mathrm{d} \xi}\left\{D \frac{\mathrm{d} C}{\mathrm{~d} \xi}\right\}
$$

in the parabolic space $\xi=x / \sqrt{t}$ [26]. The general solutions of Equation (26) had never been also reported until recently [14] [27] [28].

Diffusion experiments have been widely performed using a diffusion couple 
for the one-dimensional space. The essence of diffusion theory is still kept in even that case. In the following, therefore, the analytical theory of diffusion equation is developed in case of the space-time $(t, x)$. In that case, the normalized condition of $\sum_{i=1}^{N} C^{j}=1$ mentioned above is valid on a cross section perpendicular to the diffusion direction, as far as the shape variation of diffusion specimen is negligible as usual experiments.

In accordance with the usual analytical method of differential equation, the general solutions of equation (26) were reasonably obtained as

$$
\begin{aligned}
& D^{ \pm}(\xi)=D_{\mathrm{m}}-D_{\Delta} \operatorname{erf}\left(\frac{\xi}{2 \sqrt{D_{\mathrm{int} \pm}}}-\frac{\xi_{\mathrm{IF}}}{2 \sqrt{D_{\mathrm{int}+}}}+\operatorname{erf}^{-1}\left(\frac{D_{\mathrm{m}}-D_{\mathrm{IF}}}{D_{\Delta}}\right)\right) \\
& C^{ \pm}(\xi)=C_{\mathrm{m}}-C_{\Delta} \operatorname{erf}\left(\frac{\xi}{2 \sqrt{D_{\mathrm{int} \pm}}}-\frac{\xi_{\mathrm{IN}}}{2 \sqrt{D_{\mathrm{int}-}}}+\operatorname{erf}^{-1}\left(\frac{C_{\mathrm{m}}-C_{\mathrm{IN}}}{C_{\Delta}}\right)\right) .
\end{aligned}
$$

in the previous work [14]. Here, the notation \pm corresponds to \pm of the parabolic coordinate $\xi(=x / \sqrt{t})$, where the origin $x=0$ of a fixed coordinate system is set at the initial junction interface in a diffusion couple composed of materials $\mathrm{A}$ $(x<0)$ and $\mathrm{B}(x>0)$. The physical constants in Equations (27) and (28) determined from an initial and/or boundary values of diffusivity and concentration at $\xi=\xi_{\mathrm{A}}$ and $\xi=\xi_{\mathrm{B}}$ in the diffusion region $\xi_{\mathrm{A}} \leq \xi \leq \xi_{\mathrm{B}}$ are as follows:

$$
\begin{gathered}
D_{m}=\left(D_{\mathrm{A}}+D_{\mathrm{B}}\right) / 2, D_{\Delta}=\left(D_{\mathrm{A}}-D_{\mathrm{B}}\right) / 2, \\
C_{m}=\left(C_{\mathrm{A}}+C_{\mathrm{B}}\right) / 2, C_{\Delta}=\left(C_{\mathrm{A}}-C_{\mathrm{B}}\right) / 2, \\
\xi_{\mathrm{IF}}=0, \xi_{\mathrm{IN}}=2 \sqrt{D_{\mathrm{int}-}}\left(\sqrt{D_{\mathrm{A}}}-\sqrt{D_{\mathrm{B}}}\right) /\left(\sqrt{D_{\mathrm{A}}}+\sqrt{D_{\mathrm{B}}}\right), D_{\mathrm{IF}}=\frac{D_{\mathrm{A}}-D_{\mathrm{B}}}{\ln D_{\mathrm{A}}-\ln D_{\mathrm{B}}}, \\
C_{\mathrm{IN}}=C_{\mathrm{m}}-C_{\Delta} \frac{D_{\mathrm{m}}-D_{\mathrm{IF}}}{D_{\Delta}}, D_{\text {int }+}=\frac{D_{\mathrm{A}}+D_{\mathrm{B}}}{2}, D_{\text {int }-}=\sqrt{D_{\mathrm{A}} D_{\mathrm{B}}} .
\end{gathered}
$$

In addition, when the physical field around a diffusion particle is considered to be uniform, the diffusivity $D$ is accepted as a physical constant $D_{0}$. In that case, Equation (26) is rewritten as

$$
-\frac{\xi}{2} \frac{\mathrm{d} C}{\mathrm{~d} \xi}=D_{0} \frac{\mathrm{d}^{2} C}{\mathrm{~d} \xi^{2}} .
$$

On the other hand, the diffusivity expression of Equation (27) then becomes

$$
D_{\mathrm{A}}=D_{\mathrm{B}}=D_{0} \rightarrow D^{ \pm}(\xi)=D_{0}
$$

and the concentration profile of Equation (28) is expressed as

$$
C^{ \pm}(\xi) \rightarrow C(\xi)=C_{\mathrm{m}}-C_{\Delta} \operatorname{erf}\left(\frac{\xi}{2 \sqrt{D_{0}}}\right) .
$$

Here, Equation (30) itself is the general solution of Equation (29). Therefore, the general solution of Equation (29) is included in those of Equations (27) and (28) as an especial case. 
The uniqueness of mathematical solution of differential equation reveals that physical solutions are determined as having no alternative by substituting initial and/or boundary values into their general solutions. Therefore, if we substitute the initial and/or boundary values relevant to $j$ element for interdiffusion problems of a $N$ elements system into the general solutions of Equations (27) and (28), the physical solutions of $j$ element among $N$ elements are possible. The analytical problems of nonlinear diffusion were thus fundamentally solved. Further, when there is an inhomogeneous term in the given diffusion equation, it is necessary to obtain a particular solution relevant to the Green function for solving the differential equation, in addition to the general solutions of Equations (27) and (28).

The solutions of Equations (27) and (28) were already reported in 2011 [14]. Nevertheless, the importance has not yet been universally known to researchers. As mentioned later, there has been such a situation that misunderstanding theory is widely accepted for a long time in the existing diffusion field [24]. In the following, therefore, the application of Equations (27) and (28) to actual problems is briefly explained here.

In the diffusion experiments, we can obtain not a diffusivity profile but an only concentration profile. When the diffusivity is physically considered to be such a constant value as a case of a self-diffusion or an impurity-diffusion, we can determine the diffusivity unknown quantity by fitting Equation (30) to the concentration profile of $j$ element obtained from experiments, using the given initial concentration values for Equation (30).

In general, a diffusivity of $j$ element for a diffusion system of $L$ elements in a material A depends on $(t, x)$ in case of Equation (25). There had been no mathematical methods to determine a diffusivity of $j$ element in a diffusion system of $L$ elements. In accordance with the present method, however, $D_{\mathrm{A}}^{j}$ of $j$ element in the material A is reasonably obtained by using Equations (27), (28) and (30).

Here, we suppose experiments of a diffusion couple, where it is smoothly jointed at an initial interface between the material $A$ mentioned above and a pure material B composed of $k$ element among $L$ elements in the material A. As a matter of course, the initial value of concentration $C_{\mathrm{A}}^{j}$ of $j$ element in the material $\mathrm{A}$ is known. In that case, the diffusivity values applicable to initial and/or boundary ones in the material $\mathrm{B}$ are determined as

$$
D_{\mathrm{B}}^{1}=D_{\text {imp }}^{1}(\mathrm{~B}), \cdots, D_{\mathrm{B}}^{k}=D_{\text {self }}^{k}(\mathrm{~B}), \cdots, D_{\mathrm{B}}^{j}=D_{\text {imp }}^{j}(\mathrm{~B}), \cdots, D_{\mathrm{B}}^{\mathrm{L}}=D_{\text {imp }}^{\mathrm{L}}(\mathrm{B})
$$

by fitting Equation (30) to concentration profiles obtained from usual experiments for an impurity diffusion of $j(\neq k)$ element and a self-diffusion of $k$ element in the pure material $\mathrm{B}$, regardless of the present diffusion couple.

Subsequently, if we substitute the diffusivity $D_{\mathrm{B}}^{j}=D_{\text {imp }}^{j}(\mathrm{~B})$ or $D_{\mathrm{B}}^{k}=D_{\text {self }}^{k}(\mathrm{~B})$ obtained above to Equation (28), the only diffusivity $D_{\mathrm{A}}^{j}$ or $D_{\mathrm{A}}^{k}$ is unknown value then, because of the given initial concentration $C_{\mathrm{A}}^{j}, C_{\mathrm{B}}^{j}=0$ or $C_{\mathrm{A}}^{k}$, $C_{\mathrm{B}}^{k}=1$. By fitting Equation (28) to the experimental concentration profile of $j$ or 
$k$ in the present diffusion couple, the diffusivity value $D_{\mathrm{A}}^{j}$ or $D_{\mathrm{A}}^{k}$ is thus determined.

For a diffusion couple between the above material $\mathrm{A}$ and a new material $\mathrm{B}$ composed of $M$ elements, we suppose that there are $N(L \leq N, M \leq N)$ elements in the diffusion region. In that case, a diffusivity value $D_{\mathrm{B}}^{k}$ of $k$ element in the material $\mathrm{B}$ is also determined by the analytical method mentioned above. Therefore, if $j=k$ is valid in the diffusion region, substituting the diffusivity values $D_{\mathrm{A}}^{j}, D_{\mathrm{B}}^{k}$ determined here into Equation (27) gives a diffusivity profile in the diffusion system of $N$ elements, and also substituting those diffusivity values and the given initial and/or boundary values $C_{\mathrm{A}}^{j}, C_{\mathrm{B}}^{k}$ of concentration into Equation (28) yields a concentration profile.

If $j \neq k$ and $C_{\mathrm{A}}^{j}=0$ (or $C_{\mathrm{B}}^{k}=0$ ) is valid, by replacing the above diffusivity value $D_{\mathrm{A}}^{j}$ (or $D_{\mathrm{B}}^{k}$ ) with a diffusivity value $D_{\text {imp }}^{j}(\mathrm{~A})$ (or $D_{\text {imp }}^{k}(\mathrm{~B})$ ), which is determined from experiments for an impurity diffusion of $j$ (or $k$ ) element in the material A (or B), we can obtain the physical solutions of Equations (27) and (28).

For the analysis of interdiffusion problems, it is thus extremely important that the general solutions of Equations (27) and (28) are theoretically obtained.

4) Coordinate system of diffusion equation

Generally, there is no such a conception as a migration or a concentration for the space in physics. However, if we consider a thermodynamic influence on a material in the region $V$ within a single closed surface $S$, the expanding or the shrinking $S$ is physically conceived. In that case, the observer on the surface $S$ seems that the space migrates relatively against the observer itself and the original region $V$ changes.

For the diffusion region space except the volume of micro particles themselves in the diffusion region, the conception of a vacancy flux and a vacancy concentration is widely accepted also in the existing diffusion theory. The conception of a relative migration of diffusion region space and its rate for a whole diffusion region are thus physically accepted.

The diffusion flux $|J(t)\rangle$ of Equation (18), considered as a mathematical problem relevant to a degree of freedom resulting from applying $|J(t, x, y, z)\rangle$ dependent on time $t$ to the Gauss divergence theorem, means a relative migration of diffusion region space. When the origin of a moving coordinate system $\left(t^{\prime}, x^{\prime}, y^{\prime}, z^{\prime}\right)=\left(t^{\prime},\left|r^{\prime}\right\rangle\right)$ moves with a velocity $|v\rangle$ against that of a fixed one $(t, x, y, z)=(t,|r\rangle)$, the relation of

$$
|r\rangle=\left|r^{\prime}\right\rangle+\int_{0}^{t^{\prime}}|v\rangle \mathrm{d} t+\left|r_{0}\right\rangle
$$

is valid under the condition of $t^{\prime}=t$ and $\left|r^{\prime}\right\rangle=0,|r\rangle=\left|r_{0}\right\rangle$ at $t^{\prime}=t=0$.

Using Equation (31), the relations between differential operators in their coordinate systems are expressed as

$$
\partial_{t}=\partial_{t^{\prime}}+\left\langle\tilde{v} \mid \nabla^{\prime}\right\rangle,|\nabla\rangle=\left|\nabla^{\prime}\right\rangle .
$$

Substituting Equation (32) into Equation (4) of 


$$
\frac{\partial}{\partial t} C(t, x, y, z)=\langle\tilde{\nabla} \mid D \nabla\rangle C(t, x, y, z)
$$

expressed as a fixed coordinate system $(t, x, y, z)=(t,|r\rangle)$, the relation of

$$
\begin{aligned}
& \frac{\partial}{\partial t^{\prime}} C\left(t^{\prime}, x^{\prime}, y^{\prime}, z^{\prime}\right) \\
& =\left\langle\tilde{v} \mid \nabla^{\prime}\right\rangle C\left(t^{\prime}, x^{\prime}, y^{\prime}, z^{\prime}\right)+\left\{\left(\left\langle\tilde{\nabla}^{\prime}\right| D\right)\left|\nabla^{\prime}\right\rangle+D\left\langle\tilde{\nabla}^{\prime} \mid \nabla^{\prime}\right\rangle\right\} C\left(t^{\prime}, x^{\prime}, y^{\prime}, z^{\prime}\right) \\
& =D\left\langle\tilde{\nabla}^{\prime} \mid \nabla^{\prime}\right\rangle C\left(t^{\prime}, x^{\prime}, y^{\prime}, z^{\prime}\right)+\left\{\left\langle\tilde{v}|+\langle\tilde{\nabla}| D\} \mid \nabla^{\prime}\right\rangle C\left(t^{\prime}, x^{\prime}, y^{\prime}, z^{\prime}\right)\right.
\end{aligned}
$$

is valid.

Since the diffusion region space itself is continuous and has no mass, the relation of

$$
\langle\tilde{v}|+\left\langle\tilde{v}_{\mathrm{p}}\right|=0
$$

is physically valid between $|v\rangle$ of Equation (31) and $\left|v_{\mathrm{p}}\right\rangle$ of Equation (20). Thus, the second term in Equation (33) disappears because of $\left\langle v_{\mathrm{p}}\right|=\langle\tilde{\nabla}| D$, and Equation (33) becomes Equation (5) of

$$
\frac{\partial}{\partial t^{\prime}} C\left(t^{\prime},\left|r^{\prime}\right\rangle\right)=D\left\langle\tilde{\nabla}^{\prime} \mid \nabla^{\prime}\right\rangle C\left(t^{\prime},\left|r^{\prime}\right\rangle\right)
$$

in the moving coordinate system. As a matter of course, the inverse transformation is also possible [9] [10]. At this point, Equation (4) is now not a law but a basic equation in physics because of the theoretical derivation of Equation (5).

Rewriting Equation (33) into

$$
\frac{\partial}{\partial t^{\prime}} C\left(t^{\prime}, x^{\prime}, y^{\prime}, z^{\prime}\right)-\left\langle\tilde { \nabla } ^ { \prime } \left|\left\{|v\rangle+D\left|\nabla^{\prime}\right\rangle\right\} C\left(t^{\prime}, x^{\prime}, y^{\prime}, z^{\prime}\right)=0,\right.\right.
$$

and if we compare it with Equation (17), a diffusion flux $\left|J^{\prime}\right\rangle$ of

$$
\left|J^{\prime}\right\rangle=\left|J_{\mathrm{F}}^{\prime}\right\rangle+\left|v_{\mathrm{p}}\right\rangle C+\left|J_{\text {eq }}\right\rangle \leftarrow\left|J_{\mathrm{F}}^{\prime}\right\rangle=-D\left|\nabla^{\prime}\right\rangle C,\left|v_{\mathrm{p}}\right\rangle=-|v\rangle
$$

is obtained as a moving coordinate system, using a diffusion flux $\left|J_{\text {eq }}\right\rangle$ independent of the space-time. Further, when $|v\rangle=0\left(=-\left|v_{\mathrm{p}}\right\rangle=0\right)$ is valid in Equation (31), since the moving coordinate system $\left(t^{\prime}, x^{\prime}, y^{\prime}, z^{\prime}\right)=\left(t^{\prime},\left|r^{\prime}\right\rangle\right)$ is consistent with the fixed coordinate system $(t, x, y, z)=(t,|r\rangle)$ then, the diffusion flux of Equation (34) is rewritten as

$$
|J\rangle=\left|J_{\mathrm{F}}\right\rangle+\left|J_{\text {eq }}\right\rangle
$$

in the fixed coordinate system.

The Brown motion reveals that micro particles move randomly also under the condition of concentration gradient zero like a pure material. As far as Equation (3) is accepted as a law, the theoretical equation of diffusion flux is impossible then, because of $|\nabla\rangle C=0$. In the existing diffusion theory, therefore, the self-diffusion has been understood from diffusion phenomena of isotope elements of extremely small quantities introduced into a pure material concerned. In the strict sense of the word, however, that corresponds to a diffusion problem relevant to the impurity diffusion. 
In the present diffusion theory, the theoretical equation of self-diffusion is given by

$$
J_{x}(t, x, y, z)=-\int \partial_{t} C(t, x, y, z) \mathrm{d} x, \text { for }|J(t, x, y, z)\rangle=\left|J_{\text {eq }}\right\rangle,
$$

where $J_{x}(t, x, y, z)$ is the $x$ component of the diffusion flux $|J(t, x, y, z)\rangle$ [9] [23]. Therefore, judging from the discussion mentioned here, the diffusion flux $\left|J_{\text {eq }}\right\rangle$ is thus indispensable for understanding the diffusion theory in case of concentration gradient $|\nabla\rangle C=0$.

The well-known Kirkendall effect (K-effect $\Delta x_{\text {eff }}$ ) reveals that the jointed interface of diffusion couple shifts from the initial position to the diffusion direction in the interdiffusion problems [29]. At the same time, the phenomena indicate that the diffusion region space migrates in a diffusion region. Further, the matter also indicates that the moving and fixed coordinate systems of diffusion equation are indispensable for understanding diffusion phenomena. In the following, the formative mechanism of the K-effect is discussed.

In case of the one-dimensional space for Equation (31), using the relation of

$$
x=x^{\prime}+\int_{0}^{t^{\prime}} v_{x} \mathrm{~d} t
$$

valid between the coordinate systems under the condition of $x=x^{\prime}=0$ on the initial interface jointed at $t=t^{\prime}=0$, the problem between the coordinate systems of diffusion equations is discussed in the following.

The solution of diffusion equation corresponds to a concentration profile of the experiments carried out at a high temperature $T_{\mathrm{E}}$. On the other hand, experimental data are usually obtained at a room temperature $T_{\mathrm{R}}$. In order to compare the solution with the corresponding experimental data, therefore, it is necessary to grasp a thermal behavior of the diffusion region space between $T_{R} \leq T \leq T_{\mathrm{E}}$.

The number of diffusion particles diffusing for $x>0$ direction is generally different from one diffusing for $x<0$ direction. In other words, if vacancies are rich in the diffusion region of $x>0$ compared to their thermal equilibrium values, they are poor in the diffusion region of $x<0$, and vice versa. In the state of $T=T_{\mathrm{E}}$ just after diffusion experiments, it is considered that the coordinate origin $x^{\prime}=0$ at $t=t^{\prime}=0$ shifts from the initial position $x=0$ to $x=l_{\mathrm{E}}$ at $t=t_{\mathrm{E}}$ as given by

$$
l_{E}=\int_{0}^{t_{E}} v_{x} \mathrm{~d} t
$$

Vacancies in the vacancy rich region diffuse into the vacancy poor region during the temperature fall of $T_{E} \rightarrow T_{R}$ like the diffusion region space reaches a thermal equilibrium state. In that case, it is also considered that a quantity $Q$ of vacancies flows from a vacancy rich region to the specimen surface, because of the specimen surface of a sink of vacancies. At the same time, a quantity $Q$ of vacancies flows from the specimen surface to a vacancy poor region, because of the specimen surface of a source of vacancies then.

The formative mechanism of the K-effect depends on a material characteristic 
of a specimen used as a diffusion couple. In the above discussion, the K-effect is expressed by the relation of

$$
\Delta x_{\text {eff }}=Q / S \text {, }
$$

where $S$ is a cross section of specimen used as a diffusion couple [30] [31] [32]. In addition, the theoretical equation of the K-effect yielding

$$
\Delta x_{\text {eff }}=\alpha_{\text {eff }}(N-1) \sum_{j=1}^{N}\left\{C_{\mathrm{A}}^{j}-C_{\mathrm{B}}^{j}\right\} \sqrt{D_{\gamma}^{j} t}
$$

is valid in accordance with the parabolic law then, where the suffix $\gamma$ means $\gamma \rightarrow \mathrm{A}$ if $C_{\mathrm{A}}^{j} \geq C_{\mathrm{B}}^{j}$ or $\gamma \rightarrow \mathrm{B}$ if $C_{\mathrm{A}}^{j}<C_{\mathrm{B}}^{j}$ and $\alpha_{\text {eff }}$ is a parameter dependent on a material characteristic used for the diffusion couple [9].

\section{5) Einstein's paradox}

Using the van't Hoff law relevant to an osmostic pressure, the Stocks law in a fluid, and the Fick first law relevant to a diffusion flux, Einstein theoretically investigated behavior of the well-known Brown motion of pollen in water and he obtained the expression of diffusivity yielding

$$
D=R T / k N_{\mathrm{A}},
$$

where $R, N_{\mathrm{A}}$ and $k$ are the gas constant, the Avogadro constant and a proportional constant of an external force $|F\rangle$ used as $|F\rangle=-k\left|v_{\mathrm{p}}\right\rangle$ for a micro particle moving with a velocity $\left|v_{\mathrm{p}}\right\rangle$. As a result, it was revealed that a self-diffusion of water molecules is visualized by the behavior of pollen. In other words, it was revealed that a material is composed of such a fundamental particle as an atom and/or a molecule.

Einstein conceived then that the diffusion flux $|J\rangle$ becomes

$$
|J\rangle=\left|v_{\mathrm{p}}\right\rangle C
$$

under the condition of a mechanical equilibrium state for diffusion particles, where the diffusion particle moves with a velocity $\left|v_{\mathrm{p}}\right\rangle$ during an interaction of $|F\rangle=-k\left|v_{\mathrm{p}}\right\rangle$. Here, substituting Equation (42) into the continuous Equation (17) yields not the diffusion equation but the well-known Euler's equation in a liquid given by

$$
\partial_{t} C=-\left\langle\tilde{\nabla} \mid v_{\mathrm{p}}\right\rangle C
$$

The theoretical frame developed here by Einstein is apparently inconsistent. In the following, the Einstein's paradox is revealed as a problem of coordinate systems of diffusion equation.

There was no discussion about coordinate systems of diffusion equation in those days. The diffusion flux $|J\rangle=\left|v_{\mathrm{p}}\right\rangle C$ used here should be accepted as $\left|J^{\prime}\right\rangle=\left|v_{\mathrm{p}}\right\rangle C$ of a moving coordinate system. In that case, $\left|J_{\mathrm{F}}^{\prime}\right\rangle+\left|J_{\text {eq }}\right\rangle=0$ is valid in Equation (34) and we have $\left|J_{\mathrm{F}}^{\prime}\right\rangle=0$ then because of neglecting $\left|J_{\text {eq }}\right\rangle$ in those days. Therefore, Einstein obtained Equation (41) in such a thermal equilibrium state that the relation of $\lim _{v_{\mathrm{p}} \rightarrow 0}\left\{J_{\mathrm{F}}^{\prime} / v_{\mathrm{p}} C\right\}=1$ is valid.

In the present theory, Equation (41) is directly obtained from substituting 
$|F\rangle=-k\left|v_{\mathrm{p}}\right\rangle$ into Equation (20) because of $R=N_{\mathrm{A}} k_{\mathrm{B}}$. The present theory yields Equation (41) regardless of whether the diffusion system is in a thermal equilibrium state or not.

If we compare the present theory to a carved statue by single knife, Einstein's theory corresponds to one by assembling complicated pieces of wood. The matter discussed here gives evidence that the new diffusion theory is meaningful for a fundamental physics.

6) Historical misunderstanding problems in diffusion theory

In relation to having been no conception of a moving or a fixed coordinate system for diffusion equation, misunderstanding problems have been widely accepted in the existing diffusion theory. Further, in relation to analyzing a diffusion equation, mathematically wrong methods for solving a differential equation have been also widely accepted for a long time.

In the following, interdiffusion problems in case of $N=2$ for Equation (24) are discussed in order to reveal misunderstanding problems.

As mentioned above, it is apparent that the discussion about a moving or a fixed coordinate system for diffusion equation is indispensable for understanding diffusion theory. The K-effect affords an experimental evidence for the correlation between those coordinate systems. Nevertheless, a relation of diffusion flux, which is similar to Equation (34) of a moving coordinate system, has been widely accepted as a fixed coordinate system in the existing diffusion theory [33].

In the history of diffusion, the relation of $\tilde{D}=D^{\mathrm{I}}=D^{\mathrm{II}}$ between operators in a differential Equation (24) was misunderstood as a relation of physical diffusivity in the interdiffusion problems. If the relation $D^{\mathrm{I}}=D^{\mathrm{II}}$ is accepted as a physical diffusivity, it was then considered that understanding of the K-effect is impossible. Thereupon, the wrong conception of intrinsic diffusion was caused for interdiffusion phenomena in those days. In other words, the new intrinsic diffusivities $D_{\text {rin }}^{\mathrm{I}}, D_{\text {rin }}^{\mathrm{II}}$ satisfying the relation $D_{\text {rin }}^{\mathrm{I}} \neq D_{\text {rin }}^{\mathrm{II}}$ were thus phenomenologically incorporated into the theory of interdiffusion problems.

Based on the conception of intrinsic diffusion, Darken proposed a relation of

$$
\tilde{D}=D_{\text {rin }}^{\mathrm{I}} C^{\mathrm{II}}+D_{\text {rin }}^{\mathrm{II}} C^{\mathrm{I}} \text {, }
$$

where $\tilde{D}$ was accepted as not an operator but a physical diffusivity [34]. The so-called Darken equation has been widely used for numerical analyses of interdiffusion problems [35] [36] [37]. It was, however, reported that Equation (44) is not mathematically valid because of mathematical errors in the derivation process [24]. Before that, it is believed that such conception of an intrinsic diffusion is an illusion conceived in those days, judging from the conception of operator $\tilde{D}$ and further from the physical essence of diffusivity resulting from the derivation of diffusion equation.

The misunderstood matters relevant to the $\mathrm{K}$-effect, the intrinsic diffusion, the Darken equation, and etc. have been widely accepted for a long time in the diffusion field. In fact, they have been plausibly explained in the fundamental text- 
books [38] [39]. Misunderstanding theory in the existing diffusion field causes thus serious problems for not only researchers but also students. It is, therefore, required that the existing fundamental textbooks are suitably revised as soon as possible, also taking account of problems of coordinate systems of the diffusion equation.

\section{Discussion and Conclusions}

As far as a material is composed of micro particles, investigating behavior of those particles is indispensable for research subjects in the materials science. In that case, the Schrödinger Equation (2) has been applied to the research subjects for a few micro particles. On the other hand, the diffusion Equation (4) has been applied to research subjects for a collective motion of micro particles relevant to the Avogadro constant. Here, note that the diffusivity of diffusion equation is a physical quantity relevant to a single micro particle in a material then. It is, therefore, reasonable that the diffusivity correlates with the Schrödinger equation.

The Newton mechanics gives a source of theories in physics. In the modern physics, Einstein's relativity was established by denying the absolute time in the Newton mechanics. On the other hand, the quantum theory of another modern physics was established regardless of the Newton mechanics in those days. After that, the correlation between the quantum mechanics and the Newton mechanics has been understood as an afterthought for a long time through the correspondence principle between the physical quantity in the Newton mechanics and the corresponding mathematical operators in the quantum mechanics.

Judging from the theoretical frame in physics, it is considered that the quantum theory is still incomplete without revealing the causality for the Newton mechanics. From a viewpoint of fundamental physics, it is necessary to reveal theoretical evidence for the wave nature of an arbitrary micro particle in accordance with the cause-and-effect relationship in the Newton mechanics.

The diffusion equation having been accepted as a law for a long time since 1855 is formally transformed into the Schrödinger equation as mentioned in the text. Since the diffusion equation shows that the diffusivity depends on behavior of a micro particle in an isolated local space in a material, the transformation of the diffusion equation into the Schrödinger equation is thus reasonably accepted. It is, however, apparent that the theoretical transformation is impossible as far as we accept the Fick laws as it is. Before investigating the theoretical transformation between them, therefore, we must first grasp the physical essence of diffusivity itself.

Recently, the diffusion Equation (5) was reasonably derived from the mathematical theory of Markov process. As a result, it was first theoretically revealed that the diffusivity $D$ correlates to the angular momentum as expressed by $D=\hbar / 2 m$ for a micro particle with mass $m$ in an isolated local space. In other words, it was found that a diffusion particle makes a circuit on the surface of a local space in a material. 
On the other hand, it was also revealed that the impossibility of discrimination between two micro particles of the same kind in close vicinity to each other is equivalent to denying the mathematical density theorem of a real time in the Newton mechanics. It was thus revealed that the time $t$ in the Newton mechanics has a time $\varepsilon_{t}$ of minimum unit as a real time. In future, the conception of the time $\varepsilon_{t}$ may be accepted as a dominant conception in the fundamental physics.

As a result, it was also revealed that the differential operators $\partial / \partial t$ and $|\nabla\rangle$ in the Newton mechanics becomes $i \partial / \partial t$ and $-i|\nabla\rangle$ in the quantum mechanics. By rewriting $\partial / \partial t \rightarrow i \partial / \partial t,|\nabla\rangle \rightarrow-i|\nabla\rangle$ and $D \rightarrow \hbar / 2 m$ in the diffusion Equation (5), the Schrödinger Equation (2) is reasonably obtained. At this point, for the first time in physics, the wave nature of an arbitrary micro particle was theoretically revealed through the transformation from the diffusion equation relevant to a picture of micro particle into the wave equation of Schrödinger relevant to a wave picture. We could thus reasonably understand the necessity of quantum theory for behavior of a micro particle in accordance with the cause-and-effect relationship in the Newton mechanics.

In addition, it was also revealed that the well-known relation of matter-wave is valid as not a hypothesis but a basic equation in physics. Further, the validity of the matter-wave Equation (1) was not only reasonably revealed but also the new Equation (13) was theoretically obtained. In future, Equation (13) will be useful for understanding behavior of micro particles in a material.

Further, the derivation of diffusion equation first revealed that a moving coordinate system as well as a fixed coordinate system for the diffusion equation is essentially indispensable for understanding diffusion phenomena. The discussion about coordinate system of diffusion equation indicated that the wrong theory of diffusion has been accepted for a long time in the existing field. Concretely, the conception of intrinsic diffusion coefficient supposed to understand the Kirkendall effect is not essentially accepted judging from the basic theory of mathematical physics. In accordance with the transformation theory between those coordinate systems and the general solutions of a nonlinear diffusion equation, the new diffusion theory was reasonably established.

In history, most of laws, principles, and basic equations in physics were yielded in Europe and they have been widely accepted in the world. In such circumstances, it was revealed in the Asia country that the equations having been accepted as Fick's laws and de Broglie's hypothesis for a long time are now basic ones in physics, and further that the wave nature of an arbitrary micro particle can be reasonably explained by the conception of a time $\varepsilon_{t}$ of minimum unit as a real time in the Newton mechanics. The conception of a time $\varepsilon_{t}$ recalls the old word “刹那 (setuna)” of oriental idea to our mind then.

The basic equations shown in Figure 1 are ones described in the usual textbooks on physics. As a result, the Figure shows that the theoretical derivation of diffusion Equation (5) plays a dominant role for understanding not only a basic theory of diffusion but also a root evidence for the validity of quantum theory. 


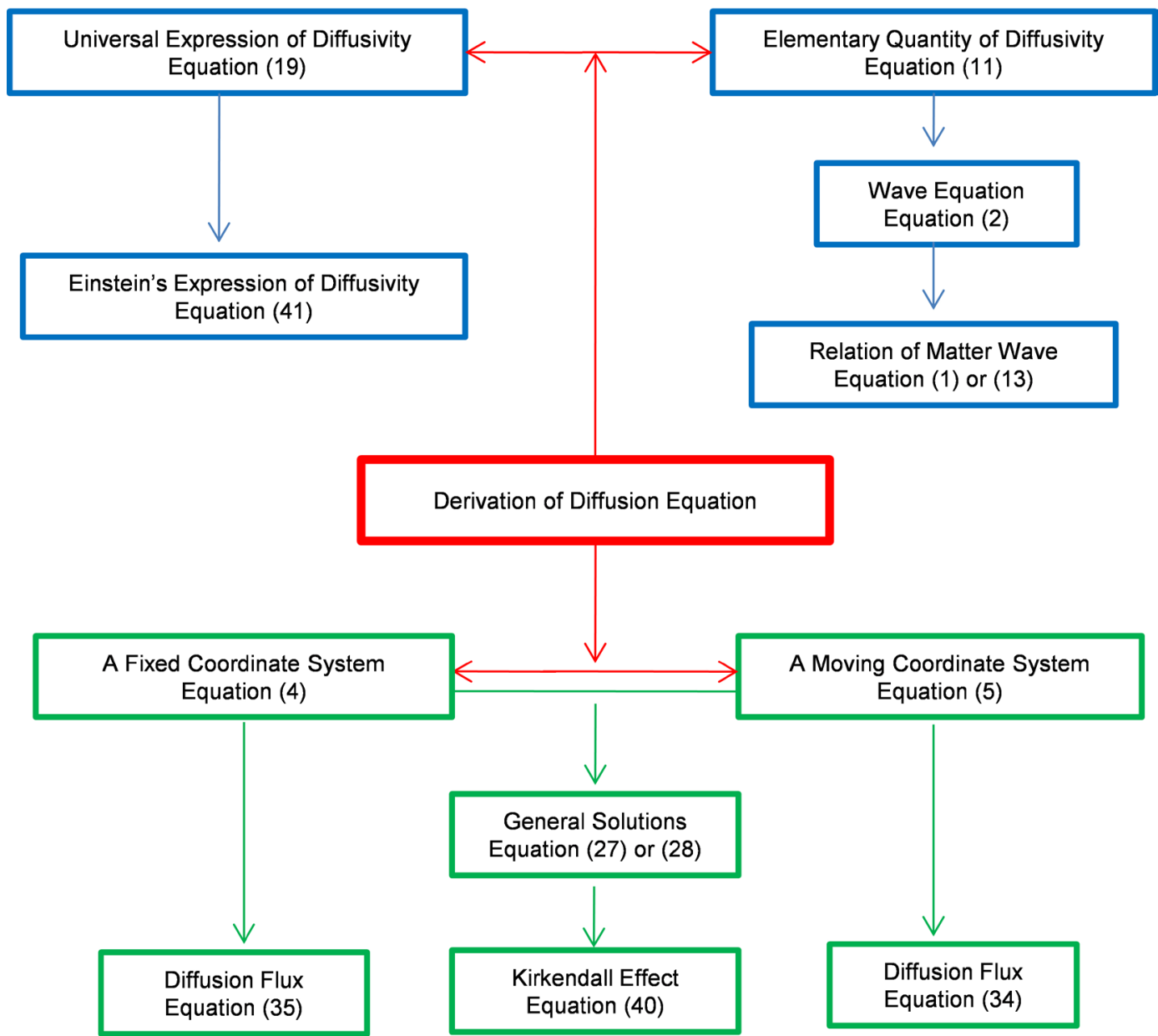

Figure 1. Basic equations resulting from derivation of diffusion equation. The basic equations, which should be accepted as new representations except the existing Equations (1), (2) and (4), are shown in the Figure. The derivation of diffusion equation is thus extremely meaningful in the fundamental physics.

From a viewpoint of the theoretical frame in physics, the matters discussed in the present paper are extremely fundamental ones as shown in the physical textbooks for students. We must have a responsibility to develop the physical truth in the textbooks. From a viewpoint of the physical education for younger people, therefore, we thus hope that researchers planning to write a fundamental textbook on physics would publish it taking account of the matters discussed above.

\section{Conflicts of Interest}

The author declares no conflicts of interest regarding the publication of this paper.

\section{References}

[1] Einstein, A. (1905) Annalen der Physik, 17, 132-148. https://doi.org/10.1002/andp.19053220607

[2] de Broglie, L. (1923) Nature, 112, 540. https://doi.org/10.1038/112540a0 
[3] Planck, M. (1900) Verhandlungen der Deutschen Physikalischen Gesellschaft, 2, 237-245.

[4] Thomson, G.P. (1927) Nature, 119, 890. https://doi.org/10.1038/119890a0

[5] Shimizu, F. (2000) Physical Review Letters, 86, 987-990. https://doi.org/10.1103/PhysRevLett.86.987

[6] Schrödinger, E. (1926) Annals of Physics, 79, 361-376. https://doi.org/10.1002/andp.19263840404

[7] Fick, A. (1855) Philosophical Magazine, 10, 31-39. https://doi.org/10.1080/14786445508641925

[8] Markov, A.A. (1960) American Mathematical Society Translations Series 2, 15, 1-14. https://doi.org/10.1090/trans2/015/01

[9] Okino, T. (2015) Journal of Modern Physics, 6, 2109-2144. https://doi.org/10.4236/jmp.2015.614217

[10] Okino, T. (2019) Applied Physics Research, 11, 82-87. https://doi.org/10.5539/apr.v11n1p82

[11] Okino, T. (2013) Journal of Modern Physics, 4, 612-615. https://doi.org/10.4236/jmp.2013.45088

[12] Okino, T. (2018) Journal of Modern Physics, 9, 1007-1028. https://doi.org/10.4236/jmp.2018.95063

[13] Okino, T. (2019) International Journal of Fundamental Physical Sciences, 9, 48-54. https://doi.org/10.14331/ijfps.2019.330131

[14] Okino, T. (2011) Materials. Transactions, 52, 2220-2227. https://doi.org/10.2320/matertrans.M2011137

[15] Boltzmann, L. (1876) Wiener Berichte, 74, 533-560.

[16] Einstein, A. (1905) Annalen der Physik, 17, 891-921. https://doi.org/10.1002/andp.19053221004

[17] Compton, A.H. (1922) Physical Review, 21, 483-503. https://doi.org/10.1103/PhysRev.21.483

[18] Einstein, A. (1905) Annalen der Phyik, 18, 549-560. https://doi.org/10.1002/andp.19053220806

[19] Langevin (1908) Comptes Rendus de Academie des Science (Paris), 146, 530-533.

[20] Balmer, J.J. (1885) Annalen der Physik, 261, 80-87. https://doi.org/10.1002/andp.18852610506

[21] Bohr, N. (1913) Philosophical Magazine, 26, 1-25. https://doi.org/10.1080/14786441308634955

[22] Frank, J. and Hertz, G. (1914) Verhandlungen der Deutschen Physikalischen Gesellschaft, 16, 357-467.

[23] Okino, T. (2012) Journal of Modern Physics, 3, 1388-1393. https://doi.org/10.4236/jmp.2012.310175

[24] Okino, T. (2013) Journal of Modern Physics, 4, 1350-1353.

[25] Boltzmann, L. (1872) Wiener Berichte, 63, 275-370.

[26] Boltzmann, L. (1894) Annual Review of Physical Chemistry, 53, 959-964. https://doi.org/10.1002/andp.18942891315

[27] Okino, T., Shimozaki, T., Fukuda, R. and Chou, H. (2012) Defect and Diffusion Forum, 322, 11-31. https://doi.org/10.4028/www.scientific.net/DDF.322.11

[28] Okino, T. (2012) Journal of Modern Physics, 3, 255-259. 
https://doi.org/10.4236/jmp.2012.33034

[29] Smigelskas, A.D. and Kirkendall, E.O. (1947) Transactions of the Metallurgical Society of AIME, 171, 130-142.

[30] Okino, T. (2014) Applied Physics Research, 6, 1-7. https://doi.org/10.5539/apr.v6n2p1

[31] Okino, T., Chou, H. and Yamada, K. (2017) Journal of Modern Physics, 8, 903-918. https://doi.org/10.4236/jmp.2017.86056

[32] Chou, H., Yamada, K. and Okino, T. (2018) Journal of Modern Physics, 9, 130-144. https://doi.org/10.4236/jmp.2018.92009

[33] Manning, J.R. (1973) Theory of Diffusion. Seminar of American Society for Metals, Metal Park Ohio, 14 October 1974, 1-23.

[34] Darken, L.S. (1948) Transactions of the Metallurgical Society of AIME, 175, 184-201.

[35] Hoshino, H., Iijima, Y. and Hirano, K. (1982) Acta Meterialia, 21, 674-682. https://doi.org/10.2320/matertrans1960.21.674

[36] Ke Haung, D., Keiser Jr., D. and Sohn, Y. (2013) Metallurgical and Materials Transactions A, 44, 738-746. https://doi.org/10.1007/s11661-012-1425-9

[37] Durand, A., Peng, L., Laplanche, G., Morris, J.R., Gerge, E.P. and Eggeler, G. (2020) Intermetallics, 122, Article ID: 106789. https://doi.org/10.1016/j.intermet.2020.106789

[38] Shewmon, P.G. (1963) Diffusion in Solids. Series in Materials Science and Engineering, McGraw-Hill, New York.

[39] Mehrer, H. (2007) Diffusion in Solid. Springer-Verlag, Berlin. 